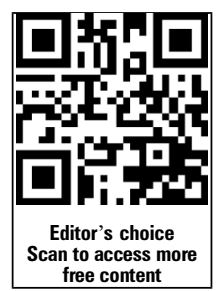

free content
- Additional material is published online only. To view please visit the journal online (http://dx.doi.org/10.1136/ heartjnl-2015-309136).

${ }^{1}$ Kaiser Permanente Northern California Division of Research, Oakland, California, USA ${ }^{2}$ San Francisco Departments of Epidemiology, University of California, Biostatistics and Medicine, San Francisco, California, USA

Division of Cardiology, Kaiser Permanente Oakland Medical Center, Oakland, California, USA

${ }^{4}$ Stanford University School of Medicine, Palo Alto, California, USA

${ }^{5}$ Center for Health Research, Kaiser Permanente Northwest, Portland, Oregon, USA

\section{Correspondence to} Carlos Iribarren, Division of Research, Kaiser Permanente Northern California, 2000 Broadway, Oakland CA 94612, USA;

Carlos.Iribarren@kp.org

Received 8 December 2015 Revised 24 February 2016 Accepted 25 February 2016 Published Online First

30 March 2016

\title{
High-sensitivity cardiac troponin I and incident coronary heart disease among asymptomatic older adults
}

\author{
Carlos Iribarren, ${ }^{1,2}$ Malini Chandra, ${ }_{1}^{1}$ Jamal S Rana, ${ }^{3}$ Mark A Hlatky, ${ }^{4}$ \\ Stephen P Fortmann, ${ }^{4,5}$ Thomas Quertermous, ${ }^{4}$ Alan S Go ${ }^{1,2,4}$
}

ABSTRACT

Objective High-sensitivity cardiac troponin I (hs-cTnl) is a novel biomarker of myocardial injury and ischaemia. Our objective was to ascertain correlates of hs-cTnl and its incremental prognostic utility for incident coronary heart disease (CHD) among older asymptomatic subjects. Methods We performed a cohort study among 1135 asymptomatic control participants in the ADVANCE (Atherosclerotic Disease, VAscular FunctioN and GenetiC Epidemiology) study at Kaiser Permanente Northern California and Stanford University, with follow-up through 31 December 2014. Hs-cTnl was measured in stored baseline (2002-2004) serum samples.

Results After a median follow-up of 11.3 years, 164 CHD events were documented. The most significant correlates of hs-cTnl were black race, body mass index, hypertension, LDL cholesterol and estimated glomerular filtration rate (eGFR) $\left(R^{2}=0.16\right)$ After adjustment for race/ethnicity, education level, diabetes status, ATP-III Framingham risk score (FRS), $C$ reactive protein and eGFR, each 1 SD increment of log-transformed Hs-cTnl was associated with $1.11(95 \% \mathrm{Cl} 1.01$ to 1.23 , $\mathrm{p}=0.04$ ) increased hazard of CHD. The c-statistic increased to 0.70 from $0.68(p=0.16)$ and the categorybased net reclassification index was $18 \%(95 \% \mathrm{Cl} 8 \%$ to $30 \%$ ) after adding hs-cTnl to the model containing the ATP-III FRS.

Conclusions Hs-cTnl conveys incremental prognostic information for incident CHD among asymptomatic older adults.

\section{INTRODUCTION}

Cardiac troponin I is a regulatory subunit of the troponin complex that interacts with the actin thin filament within cardiac muscle cells. ${ }^{1}$ Therefore, cardiac troponin I, in conjunction with cardiac troponins $\mathrm{C}$ and $\mathrm{T}$, plays an integral role in the regulation of muscle contraction. Cardiac troponin I concentrations measured with conventional assays are routinely used in the rule out of acute myocardial infarction (AMI) and in the assessment of 30 -day and 90-day prognosis among patients presenting with acute coronary syndrome (ACS). ${ }^{2}{ }^{3}$ CrossMark Recently, new generations of cardiac troponin assays ('high-sensitivity or ultra-sensitivity' troponin assays) have been developed with limits of detection at least one order of magnitude lower than current commercial assays. ${ }^{45}$

Prior studies employing high-sensitivity troponin assays, capable of measuring troponin levels in the general population or in patients with stable cardiovascular disease, have shown that elevated highsensitivity troponin levels are associated with structural heart disease, risk of future cardiovascular events and mortality. ${ }^{6-10}$ However, the additional predictive value for incident coronary heart disease (CHD) risk that high-sensitivity cardiac troponin I (hs-cTnI) may provide among asymptomatic patients has been less studied. To address this knowledge gap, we examined the distribution of hs-cTnI, its correlates, as well as the complimentary prognostic utility for incident CHD in an asymptomatic population-based sample.

\section{METHODS}

\section{Study population}

The Atherosclerotic Disease, VAscular FunctioN and GenetiC Epidemiology (ADVANCE) study was originally designed as a case-control investigation of genetic and non-genetic determinants of coronary artery disease (CAD) and mode of CAD presentation. For the present analysis, we used the control subjects with no prior cardiovascular disease derived from the adult membership of Kaiser Permanente Northern California, a large integrated healthcare delivery system providing comprehensive care to approximately a third of insured adults in the Greater San Francisco Bay Area. Kaiser Permanente members are representative of the local surrounding and state-wide insured adult population, except at the extremes of age and income distributions. These controls have been described in detail before. ${ }^{11}$ In brief, clinical and laboratory baseline data were collected between December 2001 and January 2004 among 1287 healthy men and women aged 33-69 years (as of 6 January 2001). Potential eligible participants were identified in the health plan's electronic databases, after excluding those with a major chronic disease or living over 50 miles from the research clinic. Institutional review boards of the collaborating institutions approved the study and all participants gave written informed consent prior to enrolment.

\section{Cohort derivation, study covariates and hs-cTnl laboratory methods}

All participants included in this analysis were free of CVD at baseline and attended comprehensive baseline clinic visits in 2002-2003 consisting of a physical examination (weight, height and blood
Chandra M, Rana JS, et al. 1177-1182. 
pressures by certified personnel and standard instruments and protocols), completion of a self-administered questionnaire and blood draw. Blood aliquots were banked at $-80^{\circ} \mathrm{C}$. We were unable to measure hs-cTnI in 130 subjects because they did not have stored serum specimens. Of the remaining 1157 subjects, 22 were excluded for missing variables required for estimation of the Framingham risk score (FRS) resulting in a final analysis sample of 1135 persons.

Information on age, sex, race/ethnicity, education level and smoking status was self-reported. Body mass index was estimated as weight in $\mathrm{kg}$ over height in $\mathrm{m}^{2}$. Diabetes status was derived using fasting blood glucose $\geq 7.0 \mathrm{mmol} / \mathrm{L}$, in combination with our health plan longitudinal diabetes registry. ${ }^{12}$ Hypertension was defined as self-report of hypertension or systolic blood pressure $\geq 140 \mathrm{~mm} \mathrm{Hg}$ or diastolic blood pressure $\geq 90 \mathrm{~mm} \mathrm{Hg}$ or using antihypertensive agents. Serum lipid levels (total cholesterol, high-density lipoprotein cholesterol (HDL-C) and triglycerides) were measured in fasting serum using a Beckman Synchron LX20 Analyser (Beckman Coulter, Brea, California, USA) at the Stanford Hospital and Clinics Laboratory, which is certified by the CDC/NHLBI Lipid Standardization Program. Low density lipoprotein cholesterol (LDL-C) was calculated using the Friedewald equation. ${ }^{13}$ Fasting blood glucose was determined with a hexokinase reagent kit and insulin by radioimmunoassay at Washington University, Saint Louis, Missouri, USA. Use of cholesterol-lowering agents was ascertained using electronic pharmacy dispensing records indicating an active prescription at the clinical examination date and up to 6 months prior to the clinic visit. Glomerular filtration rate (GFR) was estimated using creatine levels extracted from health plan databases and applying the Chronic Kidney Disease Epidemiology Collaboration (CKD-EPI) creatine equation. ${ }^{14}$ Plasma $\mathrm{C}$ reactive protein (CRP) was measured using an immunoturbidimetric assay on the Hitachi 917 analyser (Roche Diagnostics-Indianapolis, Indiana, USA), using reagents and calibrators from Denka Seiken (Niigata, Japan). Hs-cTnI concentrations were measured on serum specimens using the ARCHITECT STAT high-sensitivity troponin I assay (Abbott Laboratories, Abbott Park, Illinois, USA). ${ }^{5}$ This assay has within-laboratory coefficient of variation (CV) of $4.0 \%$ at the 99th centile and $\leq 0.1 \%$ cross-reactivity with skeletal troponin I and $\leq 1 \%$ cross-reactivity with cardiac troponin $\mathrm{T}$ and troponin C. The lower limit of detection (LoD) of this assay is $1.2 \mathrm{pg} /$ $\mathrm{mL} .^{5}$ Three men $(3 / 577=0.5 \%)$ and 11 women $(11 / 558=2 \%)$ had hs-cTnI below the LoD. The 99th centile of a reference population, using the same high-sensitivity assay was $23 \mathrm{pg} /$ mL. ${ }^{15}$ The ARCHITECT STAT high-sensitivity troponin I assay therefore meets the two requirements for high-sensitivity troponin assay explicitly stated by the International Federation of Clinical Chemistry: first, that the total imprecision (CV) at the 99th centile value should be $<10 \%$ and second, that measurable concentrations below the 99th centile should be attainable with an assay at a concentration value above the assay's LoD for at least 50\% (and ideally $>95 \%$ ) of healthy individuals. We estimated the 10-year risk of CHD using the Adult Treatment Panel III FRS equation that included age, sex, smoking status, systolic blood pressure, treatment for hypertension, total cholesterol and HDL cholesterol. ${ }^{16}$ We considered three risk categories, $<10 \%$ (low), $10-20 \%$ (intermediate) and $>20 \%$ (high). We did not consider the 2013 American College of Cardiology / American Heart Association (ACC/AHA) Pooled Cohort Equation $^{17}$ because it was developed including stroke and because it has been shown to overestimate cardiovascular disease (CVD) risk, particularly in Asian populations. ${ }^{18}$

\section{CHD outcome}

Incident CHD was determined from inpatient, outpatient, emergency department primary or secondary discharge diagnostic International Classification of Diseases (ICD)-9 codes consistent with non-fatal or fatal AMI (410.x), angina pectoris (411.1, 413.x), CAD (414.x), coronary revascularisation procedures (CPT4 codes 33510, 33511, 33512, 33513, 33514, 33515, 33516, 33517, 33518, 33519, 33521, 33522, 33523, 33530, 33533, 33534, 33535, 33536, 92980, 92981, 92982, 92984, 92995, 92996) or CHD death (ICD-9 codes 410-414 or ICD-10 codes I20-I25). If multiple events occurred, only the first one was counted and modelled. These codes have been previously validated by prior Kaiser Permanente of Northern California (KPNC) studies. $^{19}$

\section{Statistical methods}

We first tabulated the baseline characteristics of the cohort and then examined the bivariate association between these variables and hs-cTnI sex-specific quartiles. Differences across hs-cTnI quartiles were evaluated with analysis of variance (ANOVA) (in the case of normally distributed variables) or the Kruskal-Wallis test (in the case of skewed variables) for continuous variables and with the $\chi^{2}$ test for categorical variables. To ascertain independent correlates of hs-cTnI, we used multivariable linear regression with log-transformed hs-cTnI as the dependent variable and all study covariates as independent variables simultaneously entered in the same model. The follow-up period was determined as time from baseline to incident CHD, death by any cause identified from comprehensive mortality databases, termination of health plan membership or end of follow-up (31 December 2014), whichever occurred first. We calculated age-adjusted incidence rates of CHD per 1000 person-years with associated 95\% CIs across quartiles of hs-cTnI using Poisson regression. To examine the prospective relation of hs-cTnI with incident CHD, we employed Cox proportional hazards models with hs-cTnI as the main predictor (parametrised both as a standardised $\log _{\mathrm{e}}$-transformed continuous variable and as quartiles one through four with the first quartile as referent), first adjusting for age, sex, race/ethnicity and education level (model 1), then adjusting for model 1 covariates plus FRS (model 2), and then for model 2 covariates plus diabetes, CRP and estimated GFR (eGFR) (model 3). Formal a priori effect modification of the association between hs-cTnI and CHD by sex and FRS were examined by adding interaction terms hs-cTnI by sex and hs-cTnI by FRS, respectively. To assess the added predictive utility of hs-cTnI above and beyond FRS, we plotted the receiver operating characteristic (ROC) curves and calculated Harrel's c-statistic and integrated discrimination improvement (IDI) before and after inclusion of hs-cTnI. ${ }^{20}$ To further characterise the incremental prognostic information provided by hs-cTnI, we estimated the category-based net reclassification index (NRI), ${ }^{21}$ using standard FRS categories, namely low $(<10 \%)$, intermediate $(10-20 \%)$ and high (>20\%). Sensitivity analysis was performed using a hard CHD outcome definition that excluded angina pectoris and revascularisation procedures. All statistical analyses were done using SAS V.9.13 (SAS Institute, Cary, North Carolina, USA) and with R software. The criterion for statistical significance was a type I error $(\alpha)$ of 0.05 .

\section{RESULTS}

The mean age of the cohort was 62 years, $49 \%$ was female and about $38 \%$ was non-white (table 1). Half of the cohort had a college or higher education level and $9 \%$ reported current 
Table 1 Baseline characteristics of ADVANCE controls $(n=1135)$

\begin{tabular}{|c|c|}
\hline Age, mean (SD) & $62.1(7.7)$ \\
\hline \multicolumn{2}{|l|}{ Gender, n (\%) } \\
\hline Male & $577(50.8)$ \\
\hline Female & $558(49.2)$ \\
\hline \multicolumn{2}{|l|}{ Race/ethnicity, n (\%) } \\
\hline White & $705(62.1)$ \\
\hline Black & $101(8.9)$ \\
\hline Hispanic/Latino & $118(10.4)$ \\
\hline Asian & $103(9.1)$ \\
\hline Other/mixed & $108(9.5)$ \\
\hline \multicolumn{2}{|l|}{ Education level, n (\%) } \\
\hline Less than college & $572(50.4)$ \\
\hline College or higher & $563(49.6)$ \\
\hline \multicolumn{2}{|l|}{ Smoking status, $\mathrm{n}(\%)$} \\
\hline Never & $512(45.1)$ \\
\hline Former & $519(45.7)$ \\
\hline Current & $104(9.2)$ \\
\hline Diabetes mellitus, n (\%) & $189(16.7)$ \\
\hline Body mass index $\left(\mathrm{kg} / \mathrm{m}^{2}\right)$, mean (SD) & $28.3(5.7)$ \\
\hline Hypertension, n (\%) & $497(43.8)$ \\
\hline LDL-C (mg/dL), mean (SD) & $123.3(31.8)$ \\
\hline $\mathrm{HDL}-\mathrm{C}(\mathrm{mg} / \mathrm{dL})$, mean (SD) & $54.9(16.1)$ \\
\hline Triglycerides (mg/dL), median (IQR) & $112(79-160)$ \\
\hline Cholesterol lowering drugs, $\mathrm{n}(\%)$ & $236(20.8)$ \\
\hline $\mathrm{C}$ reactive protein (mg/dL), median (IQR) & $1.5(0.6-3.3)$ \\
\hline \multicolumn{2}{|c|}{ Estimated glomerular filtration rate $\left(\mathrm{mL} / \mathrm{min} / 1.73 \mathrm{~m}^{2}\right), \mathrm{n}(\%)$} \\
\hline$<60$ & $102(9.0)$ \\
\hline $60-89$ & $585(51.5)$ \\
\hline$\geq 90$ & $271(23.9)$ \\
\hline 10-year Framingham risk score, median (IQR) & $7.0(1.9-13.9)$ \\
\hline
\end{tabular}

smoking. The prevalence of diabetes and hypertension was $17 \%$ and $44 \%$, respectively, and $21 \%$ was on cholesterol lowering medications. The median (IQR) FRS was 7 (12), range 0.0431.9.

Hs-cTnI concentrations (in $\mathrm{ng} / \mathrm{L}$ ) ranged from 0.9 to 141 in men, and from 0.9 to 83 in women. The median hs-cTnI concentrations were $4.2 \mathrm{ng} / \mathrm{L}$ in men and $3.3 \mathrm{ng} / \mathrm{L}$ in women; the 75 th centile cut points (to define the upper quartile) were $5.5 \mathrm{ng} / \mathrm{L}$ in men and $4.2 \mathrm{ng} / \mathrm{L}$ in women, and the 99th centile cut points were $45.4 \mathrm{ng} / \mathrm{L}$ in men and $30.5 \mathrm{ng} / \mathrm{L}$ in women. The distributions of hs-cTnI in men and women, in $\log _{\mathrm{e}}$ scale, are depicted in figure 1 . Overall, there were no statistically significant differences in the distribution of education level, smoking

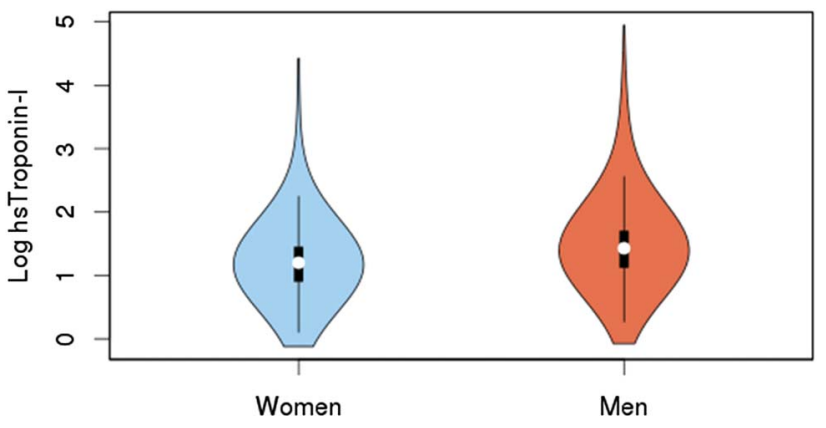

Figure 1 Violin plots showing the distribution of high-sensitivity cardiac troponin I (hs-cTnl), in $\log _{\mathrm{e}}$-scale, in men and women. status or use of cholesterol lowering drugs across sex-specific quartiles of hs-cTnI (table 2), but there were clear trends towards worse risk factor profile with increasing levels of hs-cTnI. The independent correlations of study covariates with $\log _{\mathrm{e}}$ hs-cTnI are summarised in online supplementary table S1. The most significant ones were black race $(\beta=0.30 ; \mathrm{SE}=0.06$; $\mathrm{p} \leq 0.0001$ ), body mass index ( $\beta$ per $1 \mathrm{SD}=0.10 ; \mathrm{SE}=0.02$; $\mathrm{p} \leq 0.0001)$, hypertension $(\Omega=0.14 ; \mathrm{SE}=0.04 ; \mathrm{p}=0.0002)$, LDL-C ( $($ per $1 \mathrm{SD}=0.08 ; \mathrm{SE}=0.02 ; \mathrm{p} \leq 0.001)$, eGFR $<60$ vs $\geq 90 \mathrm{~mL} / \mathrm{min} / 1.73 \mathrm{~m}^{2}(\beta=0.16 ; \mathrm{SE}=0.07 ; \mathrm{p}=0.02)$ and $\mathrm{eGFR}$ $60-89$ vs $\geq 90 \mathrm{~mL} / \mathrm{min} / 1.73 \mathrm{~m}^{2} \quad(\beta=0.09 ; \mathrm{SE}=0.04 ; \mathrm{p}=0.03)$; all together explained $16 \%$ of its variance.

The median follow-up time was 11.3 years (range, $<1-$ 13.0 years). In analysis using hs-cTnI as a standardised continuous variable in $\log _{e}$ scale, there was a significant, independent linear effect of hs-cTnI on the risk of CHD: the model $1 \mathrm{HR}$ was 1.12 (95\% CI 1.02 to 1.24 ), the model $2 \mathrm{HR}$ was 1.12 (95\% CI 1.01 to 1.24 ) and the model $3 \mathrm{HR}$ was 1.11 (95\% CI 1.01 to 1.23 ). Table 3 summarises the analysis of the association between quartiles of hs-cTnI and risk of incident CHD. In model 1, there was an almost threefold (95\% CI 1.9 to 4.9 ) increased hazard of incident CHD in the upper quartile relative to quartile 1. Further adjustments for 10-year FRS and for 10-year FRS plus diabetes, CRP and renal function mildly attenuated the strength of the association. No significant statistical interactions were found between hs-cTnI and sex $(p=0.88)$ or between hs-cTnI and FRS $(p=0.71)$, thus stratified analysis by these variables is not presented.

The ROC curves for models with and without hs-cTnI are shown in figure 2. The model containing the 10-year FRS but not hs-cTnI had a c-statistic of 0.68 ; adding hs-cTnI increased the $c$-statistic to 0.70 ( $p$-contrast $=0.16)$. The IDI was 0.0267 $(\mathrm{p}<0.0001)$. Adding hs-cTnI resulted in a category-based NRI of 0.18 (95\% CI 0.08 to 0.30$)$. In sensitivity analysis excluding angina pectoris and revascularisation procedures from the $\mathrm{CHD}$ outcome (149 events; 15 events excluded), each 1 SD increment of $\log _{\mathrm{e}}$-transformed Hs-cTnI was associated (in a fully adjusted model) with 1.12 (95\% CI 1.01 to $1.24, \mathrm{p}=0.04)$ increased hazard of CHD. The c-statistic increased to 0.69 from 0.68 $(\mathrm{p}=0.23)$ and the category-based NRI was $0.17(95 \%$ CI 0.06 to 0.30 ) after adding hs-cTnI to the model containing ATP-III FRS. The reclassification tables for non-events and events are provided as online supplementary table S2.

\section{DISCUSSION}

Supporting prior investigations, ${ }^{8} 9152223$ this biomarker substudy of ADVANCE demonstrates that circulating levels of cardiac troponin I can be detected using a high-sensitivity assay in free-living populations without prior clinical CVD. Remarkably, in our sample of older controls subjects, $100 \%$ had a measurable level of hs-cTnI. The 99th centile of hs-cTnI in our sample (45 ng/L in men and $30 \mathrm{ng} / \mathrm{L}$ in women) were higher than the 99th centile reported in a referential European cohort (MORGAM) ${ }^{24}$ and in a sample of apparently healthy volunteers in Minnesota. ${ }^{15}$ This may be due to the fact that our cohort, even though was free of clinical CVD, had a considerable risk factor burden, for example about 17\% had type- 2 diabetes, $44 \%$ had hypertension and $21 \%$ were treated for hyperlipidaemia. Also, a number of studies support the fact that occult cardiovascular disease has a significant impact on the hs-cTnI 99th centile. ${ }^{25} 26$

But more importantly, this population-based cohort study demonstrates an independent association between circulating ambulatory levels of hs-cTnI and subsequent non-fatal or fatal 
Table 2 Bivariate association of high-sensitivity cardiac troponin I with study covariates among controls $(n=1135)$

\begin{tabular}{|c|c|c|c|c|c|}
\hline & Quartile I ( $n=287$ ) & Quartile II $(n=280)$ & Quartile III $(n=286)$ & Quartile IV ( $n=282$ ) & $\mathrm{p}$ Value \\
\hline Age, mean (SD) & $62.8(6.8)$ & $62.5(7.4)$ & $60.7(8.7)$ & $62.5(7.7)$ & 0.004 \\
\hline Female, n (\%) & $141(49.1)$ & $138(49.3)$ & $142(49.7)$ & $137(48.6)$ & 1.0 \\
\hline \multicolumn{6}{|l|}{ Race/ethnicity, n (\%) } \\
\hline White & $186(64.8)$ & $179(63.9)$ & $177(61.9)$ & $163(57.8)$ & 0.01 \\
\hline Black & $15(5.2)$ & $18(6.4)$ & $27(9.4)$ & $41(14.5)$ & \\
\hline Hispanic/Latino & $32(11.2)$ & $32(11.4)$ & $26(9.1)$ & $28(9.9)$ & \\
\hline Asian & $33(11.5)$ & $27(9.6)$ & $25(8.7)$ & $18(6.4)$ & \\
\hline Other/mixed & $21(7.3)$ & $24(8.6)$ & $31(10.8)$ & $32(11.4)$ & \\
\hline \multicolumn{6}{|l|}{ Education level, n (\%) } \\
\hline Less than college & $144(50.2)$ & $131(46.8)$ & $151(52.8)$ & $146(51.8)$ & 0.5 \\
\hline College or higher & $143(49.8$ & $149(53.2$ & $135(47.2$ & $136(48.2$ & \\
\hline \multicolumn{6}{|l|}{ Smoking status, n (\%) } \\
\hline Never & $128(44.6)$ & $129(46.1)$ & $121(42.3)$ & $134(47.5)$ & 0.06 \\
\hline Former & $137(47.7$ & $133(47.5$ & $125(43.7$ & $124(44.0$ & \\
\hline Current & $22(7.7$ & $18(6.4$ & $40(14.0$ & $24(8.5$ & \\
\hline Diabetes mellitus, $n(\%)$ & $51(17.8)$ & $37(13.2)$ & $39(13.6)$ & $62(22.0)$ & 0.02 \\
\hline Body mass index $\left(\mathrm{kg} / \mathrm{m}^{2}\right)$, mean (SD) & $26.7(4.8)$ & $27.8(5.1)$ & $28.4(5.7)$ & $30.2(6.6)$ & $<0.0001$ \\
\hline Hypertension, n (\%) & $100(34.8)$ & $110(39.3)$ & $120(42.0)$ & $167(59.2)$ & $<0.0001$ \\
\hline LDL-C (mg/dL), mean (SD) & $115.8(30.6)$ & $120.9(28.6)$ & $128.3(31.5)$ & $128.2(34.6)$ & $<0.0001$ \\
\hline HDL-C (mg/dL), mean (SD) & $57.5(17.3)$ & $54.9(15.9)$ & $53.3(15.4)$ & $53.8(15.5)$ & 0.01 \\
\hline Triglycerides (mg/dL), median (IQR) & $102.0(74.0-161.0)$ & $109.0(78.5-159.5)$ & $113.5(81.0-152.0)$ & $119.5(84.0-162.0)$ & 0.07 \\
\hline Cholesterol lowering drugs, $n(\%)$ & $62(21.6)$ & $50(17.9)$ & $57(19.9)$ & $67(23.2)$ & 0.4 \\
\hline$C$ reactive protein $(\mathrm{mg} / \mathrm{dL})$, median (IQR) & $1.4(0.5-3.2)$ & $1.4(0.6-3.0)$ & $1.5(0.7-3.0)$ & $1.8(0.8-4.4)$ & $<0.01$ \\
\hline \multicolumn{6}{|c|}{ Estimated glomerular filtration rate $\left(\mathrm{mL} / \mathrm{min} / 1.73 \mathrm{~m}^{2}\right), \mathrm{n}(\%)$} \\
\hline$<60$ & $23(7.7)$ & $18(6.4)$ & $21(7.3)$ & $41(14.5)$ & 0.006 \\
\hline $60-89$ & $148(51.6)$ & $144(51.4)$ & $142(49.7)$ & $151(53.6)$ & \\
\hline $90-130$ & $66(23.0)$ & $70(25.0)$ & $69(24.1)$ & $66(23.4)$ & \\
\hline$>130$ & $4(1.4)$ & $6(2.1)$ & $8(2.8)$ & $2(0.7)$ & \\
\hline Missing & $47(16.4)$ & $42(15.0)$ & $46(16.1)$ & $22(7.8)$ & \\
\hline 10-year Framingham risk score, median (IQR) & $5.7(1.9-12.1)$ & $7.1(2.0-13.8)$ & $7.0(1.7-15.1)$ & $8.2(2.3-15.6)$ & 0.02 \\
\hline
\end{tabular}

CHD among asymptomatic patients. More specifically, subjects above the 75 th centile were at about 2.5 -fold increased risk, irrespective of traditional risk factors, CRP and renal function. Our results also provide evidence that consideration of hs-cTnI resulted in improved discriminative capacity for CHD risk assessment above and beyond baseline 10-year ATP-III Framingham risk, and in significant and clinically relevant reclassification improvement. Another finding worth noting is that black race was significantly associated with higher hs-cTnI levels, and this was not explained by the differential risk factor profile in whites compared with blacks (see online supplementary table S3). This disparity should be corroborated in other multiethnic cohorts.

Our results are in agreement with those of Zeller et $a l^{27}$ among a general middle-aged Scottish population with regard to incident cardiovascular risk prediction and reclassification. In the Prospective Investigation of the Vasculature in Uppsala Seniors (PIVUS) study among elderly community dwellers in Uppsala, Sweden, hs-cTnI levels were also higher in men than in women and were associated with all-cause mortality in both genders. $^{28}$ The findings of our analysis are also commensurate with those in the FINRISK cohort, where one (multivariate adjusted) SD of hs-cTnI was associated with 1.18 increased hazard of major acute coronary events and improved the NRI by $5.1 \% .^{29}$

In the population-based Dallas Heart Study, circulating levels of cardiac troponin $\mathrm{T}$ measured with a different high-sensitivity assay was associated with structural heart disease (left ventricular wall thickening and dilation and left ventricular systolic dysfunction by cardiac MRI) and with all-cause mortality. ${ }^{9}$ In an

Table 3 Association of high-sensitivity cardiac troponin I with incident coronary heart disease among controls ( $n=1135$; 164 events)

\begin{tabular}{lcccc}
\hline & Quartile I $(\mathbf{n}=\mathbf{2 8})$ & Quartile II $(\mathbf{n}=\mathbf{2 8 0})$ & Quartile III ( $\mathbf{n = 2 8 6 )}$ & Quartile IV ( $\mathbf{n = 2 8 2 )}$ \\
\hline Number of events, $n$ & 26 & 39 & 33 & 66 \\
Number of person-years, $n$ & 2844 & 2817 & 2821 & 2382 \\
Age-adjusted rate per 1000 person-years & 9.0 & 13.7 & 13.0 & 27.3 \\
Model 1 HRs $(95 \% \mathrm{Cl})$ & 1.00 (ref) & $1.51(0.92$ to 2.49$)$ & $1.34(0.80$ to 2.24$)$ & $2.96(1.87$ to 4.87$)$ \\
Model 2 HRs $(95 \% \mathrm{Cl})$ & 1.00 (ref) & $1.40(0.85$ to 2.30$)$ & $1.09(0.65$ to 1.84$)$ & $2.47(1.55$ to 3.93$)$ \\
Model 3 HR $(95 \% \mathrm{Cl})$ & 1.00 (ref) & $1.49(0.91$ to 2.46$)$ & $1.15(0.68$ to 1.93$)$ & $2.47(1.55$ to 3.93$)$ \\
\hline
\end{tabular}




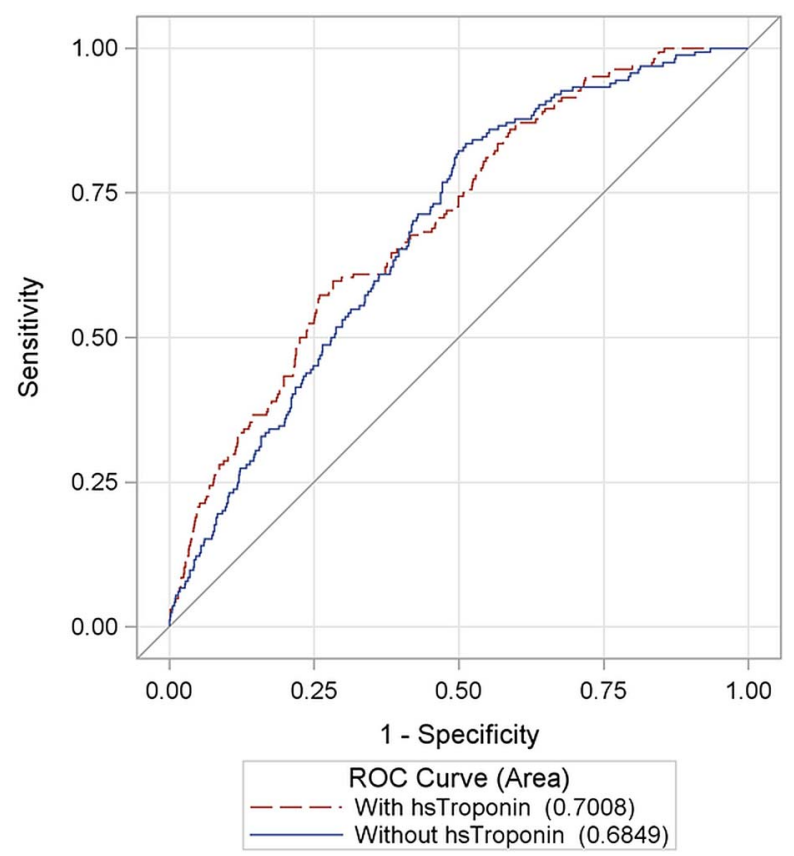

Figure 2 Receiver operating characteristic (ROC) curves for a model including baseline Framingham risk score (FRS) (solid line) and a model including baseline FRS plus high-sensitivity cardiac troponin I (hs-cTnl) (broken line).

analysis of the Cardiovascular Health Study of older adults without known heart failure, baseline and change in highsensitivity cardiac troponin $\mathrm{T}$ was significantly associated with incident heart failure and cardiovascular death. ${ }^{8}$ In the Bypass Angioplasty Revascularization Investigation in Type 2 Diabetes trial, cardiac troponin $\mathrm{T}$ concentration measured with a highsensitivity assay was an independent predictor of death from cardiovascular causes, myocardial infarction or stroke in patients with both type 2 diabetes and stable ischaemic heart disease. However, an abnormal troponin $\mathrm{T}$ value of $14 \mathrm{ng} / \mathrm{L}$ or higher failed to identify a subgroup of patients who benefited from random assignment to prompt coronary revascularisation. ${ }^{10}$ In a recent cohort study of 6304 consecutive enrolled patients with suspected ACS, those with hs-cTnI levels under $5 \mathrm{ng} / \mathrm{dL}$ had very low risk of AMI or cardiac death at 30 days (adjusted HR of $0.41,95 \%$ CI 0.21 to 0.80$).^{30}$

The strengths of the current study include the longitudinal design, the inclusion of a diverse cohort, the comprehensive and rigorous assessment of cardiovascular risk factors and the fact that the sample was derived from a real world community-based healthcare setting representative of the general insured population. However, there are limitations to keep in mind: First, the possibility of residual confounding by factors potentially associated with elevated troponin and not controlled for in the analysis, for example left ventricular hypertrophy, ${ }^{22}$ or other markers of left ventricular strain remains. Second, we could not rule out other causes of myocardial necrosis, such as myocarditis, although this is rare. Third, we lacked adequate statistical power to examine the relationship between hs-cTnI and heart failure, because only 32 patients ( 9 women, 23 men) had inpatient or outpatient utilisation consistent with heart failure during the follow-up.

In conclusion, our data provide support for the importance of subclinical ischaemia or 'troponin leak' as an independent risk factor for clinical $\mathrm{CHD}$ and inform the debate about the clinical utility of hs-cTnI assessment for risk prediction among asymptomatic subjects with no prior known cardiovascular disease. High-sensitivity troponin testing may thus play a role in screening for structural and heart disease, and for triggering more aggressive primary prevention. This represents a shift in paradigm from using troponin as a diagnostic tool in the acute setting to using it as another tool in primary prevention. Further research is needed to establish optimal cut-off thresholds for risk stratification, along with whether modification of hs-cTnI levels improves clinical outcomes.

\section{Key messages}

What is already known on this subject?

Cardiac troponin I, a marker of myocardial ischaemia, is traditionally used for the clinical diagnosis of acute myocardial infarction (AMI). Cardiac troponin measured with contemporary assays with improved sensitivity has been shown to have prognostic value for future risk of AMI in the context of unstable angina and stable coronary disease.

\section{What might this study add?}

The findings of the present study extend earlier reports of prognostic usefulness of high-sensitivity cardiac troponin I (hs-cTnl) in patients with AMI or stable coronary disease to asymptomatic persons. In particular, hs-cTnl level in the top quartile (>5.5 ng/L in men; $4.2 \mathrm{ng} / \mathrm{L}$ in women) was independently associated with 2.5 increased hazard of coronary heart disease. Furthermore, the category-based net reclassification index was 18\% (95\% Cl $8 \%$ to $30 \%$ ) after adding hs-cTnl to the model containing the ATP-III Framingham risk score.

\section{How might this impact on clinical practice?}

Findings from the study could help shift the cardiac troponin I paradigm from diagnostic utility in the acute setting to the realm of primary prevention at the population level.

Contributors Cl: Obtained funding for this study, supervised data analyses, wrote de manuscript. MC: Conducted the statistical analysis, critical review of the manuscript. JSR: Critical review of the manuscript. MAH: Critical review of the manuscript, obtained funding for the original ADVANCE study. SPF: Critical review of the manuscript, obtained funding for the original ADVANCE study. TQ: Critical review of the manuscript, obtained funding for the original ADVANCE study. ASG: Critical review of the manuscript, obtained funding for the original ADVANCE study.

Funding ADVANCE was supported by a research grant from the Donald W Reynolds Foundation. This study was also supported by Abbott Laboratories.

Competing interests $\mathrm{Cl}$ received financial support from Abbott Laboratories for this study. Abbott laboratories provided in-kind assays of hs-cTnl for this study; however, Abbott laboratory personnel were blinded to the outcome data.

Patient consent Obtained.

Ethics approval Kaiser Foundation Research Intitute Institutional Review Board. Provenance and peer review Not commissioned; externally peer reviewed.

Data sharing statement Formal requests for additional aggregate or individual risk factor and outcome data from the ADVANCE study should be made to the ADVANCE Steering Committee (all coauthors in the paper except MC and JSR).

\section{REFERENCES}

1 Perry SV. The regulation of contractile activity in muscle. Biochem Soc Trans 1979;7:593-617.

2 Morrow DA, Cannon CP, Jesse RL, et al. National Academy of Clinical Biochemistry Laboratory Medicine Practice Guidelines: clinical characteristics and utilization of biochemical markers in acute coronary syndromes. Clin Chem 2007;53:552-74. 
3 Mair J, Wagner I, Puschendorf B, et al. Cardiac troponin I to diagnose myocardial injury. Lancet 1993;341:838-9.

4 Apple FS, Collinson PO. Analytical characteristics of high-sensitivity cardiac troponin assays. Clin Chem 2012;58:54-61.

5 Krintus $M$, Kozinski M, Boudry $P$, et al. European multicenter analytical evaluation of the Abbott ARCHITECT STAT high sensitive troponin I immunoassay. Clin Chem Lab Med 2014;52:1657-65.

6 Tang WH, Wu Y, Nicholls SJ, et al. Subclinical myocardial necrosis and cardiovascular risk in stable patients undergoing elective cardiac evaluation. Arterioscler Thromb Vasc Biol 2010:30:634-40.

7 Omland T, de Lemos JA, Sabatine MS, et al. A sensitive cardiac troponin T assay in stable coronary artery disease. N Engl J Med 2009;361:2538-47.

8 deFilippi CR, de Lemos JA, Christenson RH, et al. Association of serial measures of cardiac troponin $T$ using a sensitive assay with incident heart failure and cardiovascular mortality in older adults. JAMA 2010;304:2494-502.

9 de Lemos JA, Drazner MH, Omland T, et al. Association of troponin T detected with a highly sensitive assay and cardiac structure and mortality risk in the general population. JAMA 2010;304:2503-12.

10 Everett BM, Brooks MM, Vlachos HE, et al. Troponin and cardiac events in stable ischemic heart disease and diabetes. N Engl J Med 2015;373:610-20.

11 Iribarren C, Husson G, Go AS, et al. Plasma leptin levels and coronary artery calcification in older adults. J Clin Endocrinol Metab 2007:92:729-32.

12 Karter AJ, Rowell SE, Ackerson LM, et al. Excess maternal transmission of type 2 diabetes. The Northern California Kaiser Permanente diabetes registry. Diabetes Care 1999;22:938-43.

13 Friedewald WT, Levy RI, Fredrickson DS. Estimation of the concentration of low-density lipoprotein cholesterol in plasma, without use of the preparative ultracentrifuge. Clin Chem 1972;18:499-502.

14 Levey AS, Stevens LA, Schmid $\mathrm{CH}$, et al. A new equation to estimate glomerular filtration rate. Ann Intern Med 2009;150:604-12.

15 Apple FS, Ler R, Murakami MM. Determination of 19 cardiac troponin I and T assay 99th percentile values from a common presumably healthy population. Clin Chem 2012;58:1574-81.

16 Expert Panel on Detection, Evaluation, and Treatment of High Blood Cholesterol in Adults. Executive Summary of the Third Report of The National Cholestero Education Program (NCEP) Expert Panel on Detection, Evaluation, And Treatment of High Blood Cholesterol In Adults (Adult Treatment Panel III). JAMA 2001;285:2486-97.

17 Goff DC Jr., Lloyd-Jones DM, Bennett G, et al. 2013 ACC/AHA guideline on the assessment of cardiovascular risk: a report of the American College of Cardiology/
American Heart Association Task Force on Practice Guidelines. Circulation 2014;129:\$49-73.

18 Chia YC, Lim HM, Ching SM. Validation of the pooled cohort risk score in an Asian population-a retrospective cohort study. BMC Cardiovasc Disord 2014;14:163.

19 Go AS, Chertow GM, Fan D, et al. Chronic kidney disease and the risks of death, cardiovascular events, and hospitalization. N Engl J Med 2004;351:1296-305.

20 Pencina MJ, D'Agostino RB. Overall $C$ as a measure of discrimination in survival analysis: model specific population value and confidence interval estimation. Stat Med 2004;23:2109-23.

21 Pencina MJ, D'Agostino RB Sr, D'Agostino RB Jr, et al. Evaluating the added predictive ability of a new marker: from area under the ROC curve to reclassification and beyond. Stat Med 2008;27:157-72; discussion 207-12.

22 Aeschbacher S, Schoen T, Bossard M, et al. Relationship between high-sensitivity cardiac troponin I and blood pressure among young and healthy adults. Am J Hypertens 2014;28:789-96.

23 Daniels LB, Laughlin GA, Clopton P, et al. Minimally elevated cardiac troponin T and elevated $\mathrm{N}$-terminal pro-B-type natriuretic peptide predict mortality in older adults: results from the Rancho Bernardo Study. J Am Coll Cardiol 2008;52:450-9.

24 Blankenberg S, Zeller T, Saarela 0, et al. Contribution of 30 biomarkers to 10-year cardiovascular risk estimation in 2 population cohorts: the MONICA, risk, genetics, archiving, and monograph (MORGAM) biomarker project. Circulation 2010;121:2388-97.

25 Collinson PO, Heung YM, Gaze D, et al. Influence of population selection on the 99th percentile reference value for cardiac troponin assays. Clin Chem 2012;58:219-25.

26 Koerbin G, Abhayaratna WP, Potter JM, et al. Effect of population selection on 99th percentile values for a high sensitivity cardiac troponin I and T assays. Clin Biochem 2013;46:1636-43.

27 Zeller T, Tunstall-Pedoe H, Saarela O, et al. High population prevalence of cardiac troponin I measured by a high-sensitivity assay and cardiovascular risk estimation: the MORGAM Biomarker Project Scottish Cohort. Eur Heart J 2014;35:271-81.

28 Eggers KM, Johnston N, Lind L, et al. Cardiac troponin I levels in an elderly population from the community-the implications of sex. Clin Biochem 2015; $48: 751-6$

29 Neumann JT, Havulinna AS, Zeller T, et al. Comparison of three troponins as predictors of future cardiovascular events-prospective results from the FINRISK and BiomaCaRE studies. PLoS ONE 2014;9:e90063.

30 Shah AS, Anand A, Sandoval Y, et al. High-sensitivity cardiac troponin I at presentation in patients with suspected acute coronary syndrome: a cohort study. Lancet 2015;386:2481-8. 